\title{
Upregulation of miR-128 inhibits neuronal cell apoptosis following spinal cord injury via FasL downregulation by repressing ULK1
}

\author{
RUIXUAN LIU ${ }^{1}$, ZHIBIN PENG ${ }^{1}$, YUBO ZHANG $^{1}$, RUI LI $^{2}$ and YANSONG WANG ${ }^{1}$ \\ ${ }^{1}$ Department of Orthopaedics, The First Affiliated Hospital of Harbin Medical University, Harbin, Heilongjiang 150001; \\ ${ }^{2}$ Department of Orthopaedics, Binzhou Medical University Hospital, Binzhou, Shandong 256603, P.R. China
}

Received November 9, 2020; Accepted January 21, 2021

DOI: $10.3892 / \mathrm{mmr} .2021 .12306$

\begin{abstract}
Spinal cord injury (SCI) is characterized by permanent motor deficits followed by inflammation and oxidative stress, causing neuronal cell death. The present study aimed to investigate the role of microRNA (miR)-128 in neuronal cell apoptosis and its underlying mechanism. Targeting relationships among miR-128 and Unc-51 like autophagy activating kinase 1 (ULK1) and Fas ligand (FasL) were verified using dual-luciferase reporter assay and ChIP assays. Loss- and gain-of-function assays were conducted in rat models of SCI to determine the roles of miR-128 and ULK1 in neuronal cell apoptosis, inflammation, and motor function. Apoptosis, motor function and expression of inflammatory factors were respectively determined by Terminal deoxynucleotidyl transferase-mediated dUTp nick end-labeling, Basso, Beattie and Bresnahan (BBB) score and enzyme-linked immunosorbent assay. Hematoxylin and eosin staining, Nissl staining and immunofluorescence were respectively performed to observe morphological changes and
\end{abstract}

Correspondence to: Dr Yansong Wang, Department of Orthopaedics, The First Affiliated Hospital of Harbin Medical University, 23 Youzheng Street, Nangang, Harbin, Heilongjiang 150001, P.R. China

E-mail:wys1975@163.com

Abbreviations: SCI, spinal cord injury; miR, microRNA; TUNEL, Terminal deoxynucleotidyl transferase-mediated dUTp nick end-labeling; BBB, Basso, Beattie and Bresnahan; ELISA, enzyme-linked immunosorbent assay; H\&E, Hematoxylin and eosin; ULK1, Unc-51 like autophagy activating kinase 1; LV, Lentiviral vector; sh-NC, short hairpin RNA against negative control; sh-ULK1, shRNA against ULK1; WT, wild type; MUT, mutated; oe, overexpression; ChIP, Chromatin immunoprecipitation; IgG, immunoglobulin G; RT-qPCR, reverse transcription-quantitative PCR; MTT, 3-(4,5-dimethylthiazol-2-yl)-2,5-diphenyltetrazolium bromide); FITC, fluorescein isothiocyanate; PI, phosphatidyl inositol; ANOVA, analysis of variance

Key words: spinal cord injury, apoptosis, differentiation, microRNA-128, Unc-51 like autophagy activating kinase 1, Fas ligand number of neurons and nestin-positive cells. The neuronal cells were isolated from neuron injury models and cultured in vitro. MTT and flow cytometry was conducted to determine the neuronal cell viability and apoptosis respectively. miR-128 was downregulated whereas ULK1 was upregulated in rats with SCI. Overexpression of miR-128 or downregulation of ULK1 inhibited neuronal cell apoptosis and inflammation as evidenced by an increased BBB score and more neurons and nestin-positive cells, but reduced expression of inflammatory and apoptosis-related factors. ULK1 was negatively regulated by miR-128, whereas FasL was positively regulated by ULK1. In vitro experiments validated the roles of miR-128 and ULK1 in neuronal cell differentiation and apoptosis. In conclusion, the upregulation of miR-128 depresses neuronal cell apoptosis by downregulating ULK1, thereby attenuating SCI via the downregulation of FasL.

\section{Introduction}

Spinal cord injury (SCI) is characterized by permanent motor and sensory deficits followed by secondary injury mechanisms, including prolonged inflammation and oxidative stress that leads to sustained and widespread cell death and tissue damage (1). Unfortunately, SCI remains incurable at present and the major treatments are limited to reducing secondary complications after initial traumatic injury and maximizing residual function through rehabilitation (2). Recently, cell transplantation, including neural stem cells, progenitor cells, olfactory ensheathing cells, oligodendrocyte precursor cells and mesenchymal stem cells, has been considered as a potential and viable option for the treatment of SCI (3). In addition, it has been revealed in a previous study that tanshinone IIA may enhance the therapeutic effect of bone marrow mesenchymal stem cell transplant on SCI by promoting the differentiation of neuronal cells (4). Therefore, the present study aimed to illuminate the underlying molecular mechanisms of microRNA-128 (miR-128) on the differentiation and apoptosis of neuronal cells, which demonstrated a potent ameliorating effect on SCI.

miRs, a group of non-coding small RNAs, post-transcriptionally orchestrate gene expression that has been implicated in pathogenic processes of SCI including inflammation, oxidation, demyelination, and apoptosis (5). miR-128 has been previously reported to exert a significant regulatory effect on SCI (6). miR-128 expression has been reported to 
be downregulated in cells treated with SCI, whereas the upregulation of miR-128 promotes cell viability of microglia cells in mice, thereby reducing neuropathic pain following SCI (6). Although rarely documented, Unc-51 like autophagy activating kinase 1 (ULK1) is predicted to be the downstream target gene of miR-128 through the bioinformatic website TargetScanHuman 7.2 (http://www.targetscan.org/vert_72/). As a serine/threonine kinase, ULK1 plays a key role in the regulation of autophagy activation in mice (7). The marked elevation of mRNA expression of ULK1 in clear cell renal cell carcinoma was been previously found to be associated with unfavorable clinical prognosis (8). Notably, the decreased expression of ULK1 contributes to the downregulation of goat Sertoli cell marker gene Fas ligand (FasL) (9). A previous study reported that FasL participated in the apoptosis of virus-infected cells and that the activation of FasL serves a key role in apoptosis and inflammatory response (10). The activation of FasL also exerts significant influences on apoptosis, inflammatory response, and glial proliferation in a number of nervous system diseases following SCI (11). Therefore, in this context, the purpose of the present study was to investigate the anti-apoptotic effect of miR-128 on neuronal cells following SCI by targeting the ULK1/FasL axis.

\section{Materials and methods}

Ethical statement. All animal experiments were performed following ratification by the Animal Ethics Committee of the First Affiliated Hospital of Harbin Medical University (approval no. 2019010) conforming to Principles and Procedures of the National Academy of Animal Health Care Guidelines. All efforts were made to minimize animal suffering and minimize the number of animals used.

Establishment of rat models of SCI. A total of 100 adult specified-pathogens free-grade Sprague-Dawley male rats weighing 200-220 g and 12-14 weeks old (Beijing Vital River Laboratory Animal Technology Co., Ltd.) were used in the present study. These rats were housed at $25 \pm 2{ }^{\circ} \mathrm{C}$ and humidity of $50 \pm 10 \%$ with a 12 -h light/dark cycle and given free access to water and food. Of them, 10 out of 100 rats were chosen as sham-operated rats and the remaining rats were adopted for the generation of rat models of SCI. Rats were anesthetized with $3 \%$ pentobarbital sodium via intraperitoneal injection [cat. no. P3761; Sigma-Aldrich; Merck KGaA; 30 mg/kg (1 $\mathrm{ml} / \mathrm{kg}$ according to body weight)]. Following dissection of the paravertebral muscles, laminectomy was performed on the spinal segment (T9-T11). Subsequently, rats with SCI were subjected to an aneurysm clip at the T10 level for $60 \mathrm{sec}$. Subsequent to trauma, SCI rats immediately underwent intrathecal injection with lentiviral vectors containing miR-128 and ULK 1 at the dose of $10 \mu \mathrm{l}$ and $2 \mathrm{nmol}$ (12). Finally, the incision was sutured layer by layer with silk sutures. Sham-operated rats only underwent laminectomy. All rats were injected with penicillin and analgesics for 3 days post-operation and then received manual bladder expression thrice a day. The success rate of modeling was $89 \%$ and no sham-operated rats succumbed (the remaining mice were used for experimental reserve). Following successful modeling, rats experienced spinal cord hemorrhage, delayed hindlimb extension and tail swing. Rats received manual bladder expression once a day until bladder function was restored. The Basso, Beattie and Bresnahan (BBB) scale of rats was performed on miR-128 treated on days 1, 7, 14, 21 and 28 following SCI. At the 28th day post-operation with the completion of behavioral tests, $0.2 \mathrm{ml}$ whole blood was obtained from each rat and the rats were euthanized by asphyxia with $25 \%$ capacity volume/min of $\mathrm{CO}_{2}$ gas displacement rate. The spinal cord tissues were harvested for the related tests. Lentiviral vectors (LVs) including LV-control, LV-miR-128, LV-sponge miR-128 (lentiviral vector-mediated inhibition of miR-128), short hairpin RNA against negative control (sh-NC) and shRNA against ULK1 (sh-ULK1) were constructed by Genomeditech.

$B B B$ scale. The hindlimb motor function of rats was evaluated on days $1,7,14,21$ and 28 post-operation using the BBB scoring system $(13,14)$. In an open field $(125 \mathrm{~cm}$ x $125 \mathrm{~cm})$, after rats adapted to the environment, the locomotor function was observed and scored (5 min). The observation was performed in a double-blinded manner with two trained and non-experimental staff. A total of three average recorded values were taken as the BBB score. $\mathrm{BBB}$ was a system that followed the recovery of hindlimb function from a score of 0 (no observed hindlimb movements), to a score of 21 (a normal ambulating rodent).

Dual-luciferase reporter gene assay. Bioinformatic websites RAID version 2.0 (http://www.rna-society.org/raid2/), miRDB version 5.0 (http://www.mirdb.org/miRDB/download. html), TargetScanHuman version 7.2 (http://www.targetscan. org/vert_72/) and miRWalk version 2021 (http://mirwalk. umm.uni-heidelberg.de/) were employed to predict the targeted relationship among miR-128, ULK1 and FasL. Venn map online website (http://bioinformatics.psb.ugent.be/webtools/Venn) was adopted for the validation of predicted results. The dual-luciferase reporter gene assay was employed to verify the targeting relationship between miR-128 and ULK1, as well as between ULK1 and FasL. Briefly, pmirGLO firefly luciferase-expressing vectors (Promega Corporation) was used to construct the wild type (WT) and complementary sequence mutated (MUT) site with miR-128 (PGLO-ULK1 WT and PGLO-ULK1 MUT). FasL WT and MUT (PGLO-FasL WT and PGLO-FasL MUT) and ULK1 overexpression (oe) vector and the corresponding $\mathrm{NC}$ vector were constructed. The reporter plasmids were co-transfected with the plasmids containing oe and NC of miR-128 and ULK1 into 293T cells respectively using the Lipofectamine ${ }^{\circledR} 2000$ transfection reagent (Invitrogen; Thermo Fisher Scientific, Inc.) based on the manufacturer's protocol. At $48 \mathrm{~h}$ post-transfection, Cells were lysed and centrifuged at $6,500 \mathrm{x}$ g for $1 \mathrm{~min}$ at $4^{\circ} \mathrm{C}$, followed by harvesting of the supernatant. The dual-luciferase reporter assay system (E1910, Promega Corporation) was employed to detect the luciferase activity. Relative luciferase activity=firefly luciferase/Renilla luciferase.

Chromatin immunoprecipitation (ChIP) assay. The enrichment of FasL by ULK1 was evaluated by a ChIP kit (EMD Millipore). Briefly, $1 \%$ formaldehyde was added to fix the $70-80 \%$ confluent cells at room temperature for $10 \mathrm{~min}$ to allow the formation of DNA-protein cross-links in the cells. 
Subsequently, cells were lysed in $1 \mathrm{ml}$ lysate buffer. Next, sonication (10 sec per time) was performed at intervals of $10 \mathrm{sec}$ ( 15 cycles) to fractionate large fragments into appropriate size, followed by centrifugation at $6,500 \mathrm{x}$ g for $1 \mathrm{~min}$ at $4^{\circ} \mathrm{C}$. The supernatant was then collected, divided into three tubes and then added with the positive control antibody RNA polymerase II (cat. no. ab36010; 1:1,000; Abcam), the $\mathrm{NC}$ antibody of normal mouse immunoglobulin $\mathrm{G}$ (IgG; cat. no. ab6789; 1:1,000; Abcam), or the target protein-specific rabbit anti-ULK1 antibody (cat. no. ab2851; Abcam), followed by overnight incubation at $4^{\circ} \mathrm{C}$. Protein Agarose/Sepharose was supplemented to precipitate the endogenous DNA-protein complex. Subsequent to transient centrifugation at $6,500 \mathrm{xg}$ for $1 \mathrm{~min}$ at $4^{\circ} \mathrm{C}$, the supernatant was discarded and the non-specific complexes were washed. The complex was de-crosslinked at $65^{\circ} \mathrm{C}$ and the DNA fragment was recovered by phenol/chloroform extraction and purification. The binding of ULK1 to the FasL promoter region was assessed by the specific primers in FasL promoter region using western blotting as detailed in the following section.

Hematoxylin and eosin $(H \& E)$ and Nissl staining. Spinal cord tissue located at $0.5 \mathrm{~cm}$ above and below the injury was attained 28 days post-surgery. The spinal cord tissues were fixed in $4 \%$ neutral paraformaldehyde (PFA) for $24 \mathrm{~h}$ at room temperature, routinely dehydrated with graded ethanol series (100\% for $5 \mathrm{~min}, 95 \%$ for $3 \mathrm{~min}, 90 \%$ for $3 \mathrm{~min}$ ), cleared using xylene and, embedded in paraffin, and sectioned into $4 \mu \mathrm{m}$-thick sections. The sections were then dewaxed twice with xylene (each time for $5 \mathrm{~min}$ ) and rehydrated, followed by 5 -min hematoxylin staining and 20 -sec differentiation with hydrochloric acid alcohol. Subsequent to 5-min $1 \%$ eosin staining, morphological changes in spinal cord tissue structure were observed under a light microscope (magnification, x200; Olympus BX51; Olympus Corporation).

For Nissl staining the sections were stained with $1 \%$ toluidine blue staining solution for $10 \mathrm{~min}$, followed by conventional dehydration using gradient ethanol and xylene clearing and neutral resin mounting. Quantitative analysis of the number of surviving neurons was implemented under a light microscope (magnification, x200; Olympus BX51; Olympus Corporation). In all $\sim 3-5$ rats were randomly attained in each group for statistical analysis.

Immunofluorescence. The frozen sections were air-dried at room temperature, fixed with $-20^{\circ} \mathrm{C}$ pre-chilled acetone for $6 \mathrm{~min}$ and water-bathed with $50 \%$ formamide $/ 2 \mathrm{X}$ citrate hybridization solution at $65^{\circ} \mathrm{C}$ for $2 \mathrm{~h}$. Subsequently, the sections were washed using $2 \mathrm{X}$ saline sodium citrate solution for 3 times, followed by 30 -min immersion in $0.3 \%$ Triton at ambient temperature. The sections were washed in $2 \mathrm{M} \mathrm{HCl}$ and finally washed twice in $0.1 \mathrm{~mol} / 1$ boric acid buffer $(\mathrm{pH} 8.0$; $5 \mathrm{~min} /$ time). Finally, the sections were blocked with $10 \%$ sheep serum at $37^{\circ} \mathrm{C}$ for $1 \mathrm{~h}$. Each section was then added dropwise with neuronal nuclear antigen (cat. no. ab128886), neurofilament-200 (cat. no. ab82259), glial fibrillary acidic protein (1:100; cat. no. ab7260; all from Abcam) for overnight incubation at $4^{\circ} \mathrm{C}$. The $0.01 \mathrm{M}$ phosphate-buffered saline instead of the primary antibody was adopted as a $\mathrm{NC}$. IgG was then added dropwise to the sections for 45-min incubation at room
Table I. The primer sequences used in reverse transcriptionquantitative PCR.

\begin{tabular}{ll} 
Target gene & \multicolumn{1}{c}{ Primer sequence (5'-3') } \\
\hline miR-128 & F: 5'-GGTCACAGTGAACCGGTC-3' \\
& R: 5'-GTGCAGGGTCCGAGGT-3' \\
ULK1 & F: 5'-CCCCAACCTTTCGGACTT-3' \\
& R: 5'-CCAACAGGGTCAGCAAACTC-3' \\
U6 & F: 5'-GCGCGTCGTGAAGCGTTC-3' \\
& R: 5'-GTGCAGGGTCCGAGGT-3' \\
GAPDH & F: 5'-CATCACTGCCACCCAGAAGA-3' \\
& R: 5'-TCCACGACCGACACGTTG-3'
\end{tabular}

miR-128, microRNA-128; ULK1, unc-51 like kinase 1; U6, U6 small nuclear RNA; F, forward; R, reverse.

temperature. The sections were mounted with anti-fluorescent quencher and observed under Leica SP5 confocal microscope (magnification, x 200; Leica Microsystems GmbH).

Terminal deoxynucleotidyl transferase-mediated dUTp nick end-labeling (TUNEL) staining. A one-step TUNEL apoptosis assay kit (Roche Diagnostics $\mathrm{GmbH}$ ) was employed to determine the morphological changes of cell apoptosis 28 days post-operation. Apoptotic cells were TUNEL positive in a Nikon ECLIPSE Ti inverted microscope (Nikon Corporation) and the nuclei of apoptotic cells were stained brownish-yellow. A total of 10 high-power fields were randomly observed, followed by calculation of the mean number of positive cells using BI-2000 image-analysis system (BI-2000; Visitech Systems).

Reverse transcription-quantitative (RT-q) PCR. Total RNA extraction from spinal cord tissues of rats from each group was implemented as per the instructions of TRIzol ${ }^{\circledR}$ (Thermo Fisher Scientific, Inc.). All primers were synthesized by Augct Biotechnology Co., Ltd. (Table I) according to the manufacturer's protocols. Fluorescence quantitative PCR was performed in accordance with the protocols of SYBR Premix Ex TaqTM II kit (cat. no. RR820A; Xingzhi Biotech, Co. Ltd.). The reaction system was $50 \mu \mathrm{l}$ : SYBR ${ }^{\circledR}$ Premix Ex Taq II (2X) $25 \mu \mathrm{l}$, PCR upstream primer $2 \mu \mathrm{l}$, PCR downstream primer $2 \mu \mathrm{l}$, ROX reference dye (50X) $1 \mu \mathrm{l}$, DNA template $4 \mu \mathrm{l}$, and $\mathrm{ddH}_{2} \mathrm{O} 16 \mu \mathrm{l}$. PCR was performed on ABI PRISM 7300 (Applied Biosystems; Thermo Fisher Scientific, Inc.). The reaction conditions were as follows: $10 \mathrm{~min}$ of pre-denaturation at $94^{\circ} \mathrm{C}, 15 \mathrm{sec}$ of denaturation at $94^{\circ} \mathrm{C}$ and $30 \mathrm{sec}$ of annealing at $55^{\circ} \mathrm{C}$. After 40 cycles, amplification was performed for $1 \mathrm{~min}$ at $72^{\circ} \mathrm{C}$. The relative expression was calculated using the $2^{-\triangle \Delta C q}$ method (15) and standardized by U6 (for miR-128) and GAPDH (for ULK1; cat. no. abs830032; Absin Bioscience, Inc.). The experiment was performed three times.

Western blot analysis. The total protein content was extracted from the tissue according to the manufacturer's protocols provided by high-efficiency radioimmunoprecipitation assay-like buffer (cat. no. R0010, Beijing Solarbio Science 
\& Technology Co., Ltd.). The estimation of protein concentration was implemented using a bicinchoninic acid kit (cat. no. 20201ES76; Shanghai Yeasen Biotechnology Co., Ltd.). The protein was uploaded (30 $\mu \mathrm{g}$ per well), separated by $10 \%$ polyacrylamide gel electrophoresis, and electro-blotted onto a polyvinylidene fluoride membrane. Subsequent to 1-h membrane blocking with $5 \%$ bovine serum albumin (Sigma-Aldrich; Merck KGaA) at ambient temperature, overnight membrane incubation was performed at $4^{\circ} \mathrm{C}$ with diluted primary antibodies to Cleaved caspase-3 (cat. no. ab2302; 1:1,000), FAS cell surface death receptor (Fas) (cat. no. ab82419; 1:1,000), FasL (cat. no. ab15285; 1:1,000) and GAPDH (cat. no. ab181602; 1:1,000). The membrane was washed thrice with Tris-buffered saline with $0.05 \%$ Tween 20 , each time for $5 \mathrm{~min}$ and subsequently re-probed with horseradish peroxidase-tagged goat anti-rabbit IgG (cat. no. ab150077; 1:10,000 Abcam). An electrogenerated chemiluminescence (ECL) developer (Shanghai Lianshui Biotechnology Co., Ltd.) was adopted to develop the membrane. ImageJ $1.48 \mathrm{u}$ software (National Institutes of Health) was applied for the quantitative protein analysis. Relative protein expression was analyzed by the ratio of the gray value of the target band to that of GAPDH.

Enzyme-linked immunosorbent assay (ELISA). The $0.2 \mathrm{ml}$ orbital whole blood was attained from each rats and centrifuged at $1,500 \mathrm{x} \mathrm{g}$ at room temperature for $10 \mathrm{~min}$ to collect the serum of rats. ELISA was performed in accordance with the manufacturer's protocols (cat. no. ER2101; Wuhan Msk Biotechnology Co., Ltd.) and a standard curve was drawn to determine the levels of inflammatory factors IL-1 $\beta$, IL- 6 and TNF- $\alpha$ in the serum of rats.

Neuron injury models. Primary spinal cord neurons were isolated from healthy SPF-level SD rats (Beijing Vital River Laboratory Animal Technology Co., Ltd.) on day 14-17 after gestation. Briefly, spinal cords were dissected in medium without serum and trypsinized $(0.125 \%)$ at $37^{\circ} \mathrm{C}$ for $15 \mathrm{~min}$. The cell suspension was prepared and incubated into 24-well plates coated with $0.1 \mathrm{~g} / 1$ polylysine at a cell density of $4 \times 10^{5}$ cells $/ \mathrm{ml}$ under a humidity-saturated environment with $5 \% \mathrm{CO}_{2}$ at $37^{\circ} \mathrm{C}$. The medium was renewed every 2 days. On the 3rd day of culture, cytarabine was supplemented at a final mass concentration of $5 \mathrm{mg} / \mathrm{l}$ to inhibit the growth of mixed cells. Following 5 days of culture in medium without serum, the neuronal cells were treated with $30 \mathrm{~mm} \mathrm{H}_{2} \mathrm{O}_{2}$ for $24 \mathrm{~h}$ to construct neuron injury models. Then, $24 \mathrm{~h}$ before modeling, cells were transfected with inhibitor NC (25 nM), miR-128 inhibitor (25 nM), oe-NC (25 nM), or oe-ULK1 $(25 \mathrm{nM})$ for $48 \mathrm{~h}$ using the HG transgene reagent (Genomeditech), respectively The above vectors were all constructed by Genomeditech. The sequences are shown in Table II. The neuronal cells were then incubated for $48 \mathrm{~h}$ after transfection for subsequent detection.

3-(4,5-Dimethylthiazol-2-yl)-2,5-diphenyltetrazolium bromide) (MTT) assay. Cells were prepared into $1 \times 10^{4}$ cell $/ \mathrm{ml}$ cell suspension using Dulbecco's modified Eagle's medium containing $10 \%$ fetal bovine serum (BioSer) and cultured in a 96-well plate. Then, 8 wells ( $100 \mu \mathrm{l})$ were set for each group and culture was implemented in a $37^{\circ} \mathrm{C}$ cell incubator with $5 \% \mathrm{CO}_{2}$. Subsequent to 24 -h culture, the plate was removed and $10 \mu \mathrm{l}$
MTT (Sigma-Aldrich; Merck KGaA) was added into each well for 2-h culture. The optical density value at $570 \mathrm{~nm}$ was read using an enzyme-linked immunoassay (cat. no. NYW-96M; Beijing. Noahway Instruments Co., Ltd.). The cell survival rate was calculated as follows: Cell survival rate $=($ experimental group OD-blank group OD)/(control group OD-blank group OD) $\times 100 \%$.

Flow cytometry. Cells were trypsinized in the absence of ethylene diamine tetraacetic acid and attained into a flow tube. Subsequent to cell centrifugation, the supernatant was discarded. As per the protocols of Annexin V-fluorescein isothiocyanate (FITC) apoptosis detection kit (cat. no. C1065; Beyotime Institute of Biotechnology), Annexin V-FITC, phosphatidyl inositol (PI), hydroxyethyl piperazine ethylsulfonic acid buffer was added to Annexin V-FITC/PI staining solution in the proportion of 1:2:50. Cells at a concentration of $1 \times 10^{6}$ cells per $100 \mu \mathrm{l}$ of the staining solution were resuspended, incubated at ambient temperature for $15 \mathrm{~min}$ in the dark and added with $1 \mathrm{ml}$ of 4-(2-hydroxyethyl)-1-piperazineëthanesulfonic acid buffer. Flow cytometer (Bio-Rad ZE5; Bio-Rad Laboratories, Inc.) was employed to determine the excitation wavelength at $488 \mathrm{~nm}$. The excitation wavelength at 525 or $620 \mathrm{~nm}$ was employed to detect FITC or PI fluorescence for cell apoptosis, respectively. The samples $(n=10)$ were randomly selected in each group. Apoptosis index $(\mathrm{AI})=$ number of apoptotic cells/(number of apoptotic cells + normal cells).

Statistical analysis. Statistical analysis was performed using SPSS 21.0 (IBM Corp.). Measurement data were summarized by mean \pm standard deviation or inter-quartile range. An unpaired $t$-test was performed for the variance of data consistent with the normal distribution and homogeneity of variance. One-way analysis of variance (ANOVA) was conducted for comparison among multiple groups, followed by Tukey's post-hoc test. Data at different time-points were compared using repeated-measures ANOVA, followed by Bonferroni post-hoc test. $\mathrm{P}<0.05$ was considered to indicate a statistically significant difference.

\section{Results}

miR-128 is downregulated following SCI. Rat models with SCI were induced and the BBB score was calculated from the normal rats and SCI rats on day 1, 3, 5, 7 and 14 after modeling (Fig. 1A). A markedly lower BBB score was seen in rats with SCI when compared with normal rats. The results of H\&E staining (Fig. 1B) showed that there were no significant pathological changes in normal rats, whereas rats with SCI exhibited distinct white matter edema in the spinal cord, microvascular rupture or swelling in the gray matter with focal bleeding, degeneration, and necrosis. Nissl staining (Fig. 1C) and immunofluorescence (Fig. 1D) indicated that neurons and nestin-positive cells were both markedly reduced in rats with SCI compared to normal rats. ELISA (Fig. 1E) demonstrated that the levels of IL- $1 \beta$, IL- 6 and TNF- $\alpha$ in serum notably rose in rats with SCI in comparison with those in normal rats. TUNEL results (Fig. 1F) revealed that apoptotic cells in rats with SCI were significantly increased in rats with SCI in comparison with those in normal rats. Meanwhile, western blot 
Table II. Gene inhibitor and overexpression sequence.

Group

Sequence

Inhibitor NC

miR-128 inhibitor

oe-NC

oe-ULK1
ACACUGCUGGGAAAGGUAGGG

AGUGUCACUUGGCCAGAGAAA

GAATTCCACCACACTGGACTAGTGGATCC

ATGGAGCCGGGCCGCGGCGGCGTCGAGACCGTGGGCAAGTTCGAGTTCTCTCGCAAGGACC TGATTGGACACGGCGCCTTCGCGGTGGTCTTCAAGGGTCGACACCGCGAGAAGCACGACC TGGAGGTGGCCGTCAAATGCATTAACAAGAAGAACCTTGCCAAGTCCCAAACACTGCTGG GAAAGGAAATCAAAATCCTGAAGGAACTAAAGCACGAAAACATCGTGGCGCTGTATGACTTCC AGGAAATGGCTAATTCTGTCTACCTGGTCATGGAGTATTGTAATGGTGGAGACCTGGCTGACT ACCTGCACACTATGCGCACACTGAGTGAAGACACTGTCAGGCTTTTCCTACAGCAGATCGCTG GCGCCATGCGGCTGCTGCACAGCAAGGGCATCATCCACCGGGACCTGAAGCCCCAAAACATCC TGCTGTCCAACCCTGGGGGCCGCCGGGCCAACCCCAGCAACATCCGAGTCAAGATTGCTGACT TTGGATTCGCTCGGTACCTCCAGAGCAACATGATGGCGGCCACACTCTGTGGTTCTCCTATGT ACATGGCTCCTGAGGTCATTATGTCCCAGCACTACGATGGAAAGGCTGACCTGTGGAGCATTG GCACCATTGTCTACCAGTGTCTGACAGGGAAGGCCCCTTTTCAGGCCAGCAGCCCTCAGGATT TGCGCCTGTTTTATGAGAAGAACAAGACACTAGTTCCTGCCATCCCCCGGGAGACATCAGCTC CCCTGCGGCAGCTGCTCCTGGCTCTGTTGCAGCGGAACCACAAGGACCGCATGGACTTTGATG AATTTTTCCACCACCCTTTCTTGGATGCCAGCACCCCCATCAAGAAATCCCCACCTGTGCCTG TGCCCTCATATCCAAGCTCAGGGTCTGGCAGCAGCTCCAGCAGCAGCTCTGCCTCCCACCTGG CCTCTCCACCGTCCCTGGGGGAGATGCCACAGCTACAGAAGACCCTTACCTCCCCAGCCGATG CTGCTGGCTTTCTTCAGGGCTCCCGGGACTCTGGTGGCAGCAGCAAAGACTCCTGTGACACAG ATGACTTTGTCATGGTCCCAGCCCAGTTTCCAGGTGATCTAGTTGCTGAGGCAGCCAGTGCCA AGCCCCCACCTGATAGCCTGCTGTGTAGTGGGAGCTCATTGGTGGCCTCTGCTGGCCTAGAGA GCCACGGCCGTACCCCCTCTCCCTCTCCGACCTGCAGCAGCTCTCCCAGCCCCTCTGGCCGGC CTGGCCCCTTCTCCAGCAACAGGTACGGTGCCTCGGTCCCCATTCCTGTCCCCACTCAGGTGC ACAATTACCAGCGCATCGAGCAAAACCTGCAATCGCCCACTCAACAGCAGACAGCCAGGTCCT CTGCCATCCGAAGGTCAGGGAGCACCAGCCCCCTGGGCTTTGGCCGGGCCAGCCCATCACCCC CCTCCCACACCGATGGAGCCATGCTGGCCAGGAAGCTGTCACTTGGAGGTGGCCGTCCCTACA CACCTTCTCCCCAAGTGGGAACCATCCCAGAGCGACCCAGCTGGAGCAGAGTGCCCTCCCCAC AAGGAGCTGATGTGCGGGTTGGCAGGTCACCACGACCCGGTTCCTCTGTGCCTGAGCACTCTC CAAGAACCACTGGGCTGGGCTGCCGCCTGCACAGTGCCCCTAACCTGTCCGACTTCCATGTTG TGCGTCCCAAGCTGCCTAAGCCCCCAACAGACCCACTGGGAGCCACCTTTAGCCCACCCCAGA CCAGCGCACCCCAGCCATGCCCAGGGCTACAGTCTTGCCGGCCACTGCGTGGCTCACCTAAGC TGCCTGACTTCCTACAGCGGAGTCCCCTACCCCCCATCCTAGGCTCTCCTACCAAGGCCGGGC ССТССТTTGACTTCCCCAAAACCCCCAGCTCTCAGAATTTGCTGACCCTGTTGGCTAGGCAGG GGGTAGTAATGACACCACCTCGGAACCGTACACTGCCTGACCTCTCCGAGGCCAGTCCTTTCC ATGGCCAGCAGCTGGGCTCTGGCCTTCGGCCCGCTGAAGACACCCGGGGTCCCTTTGGACGGT CCTTCAGCACCAGCCGCATTACGGACCTGCTGCTTAAGGCTGCATTTGGGACTCAGGCCTCTG ACTCAGGCAGCACAGACAGCCTACAGGAGAAACCTATGGAGATTGCTCCCTCTGCTGGCTTTG GAGGGACTCTGCATCCAGGAGCTCGTGGTGGAGGGGCCAGCAGCCCAGCACCTGTGGTATTTA CTGTAGGCTCCCCACCCAGTGGTGCCACCCCACCCCAGAGTACCCGTACCAGAATGTTCTCAG TGGGCTCTTCCAGCTCCCTGGGCTCTACTGGCTCCTCCTCTGCCCGCCACTTAGTGCCTGGGG CCTGTGGAGAGGCCCCGGAGCTTTCTGCCCCAGGCCACTGCTGTAGCCTTGCTGACCCCCTTG CTGCCAACTTGGAGGGGGCTGTGACCTTCGAGGCTCCTGACCTCCCAGAGGAGACCCTCATGG AGCAAGAGCACACGGAAACCCTACACAGTCTGCGCTTCACACTAGCGTTTGCACAGCAAGTTC TGGAGATTGCAGCCCTGAAGGGAAGTGCCAGTGAGGCCGCCGGTGGCCCTGAGTACCAGC TCCAGGAAAGTGTGGTGGCTGACCAGATCAGTCAGTTGAGCCGAGAGTGGGGCTTTGCAG AGCAACTGGTTCTGTACTTGAAGGTGGCTGAGCTGCTGTCCTCAGGCCTACAGACTGCCATTG ACCAGATTCGAGCTGGCAAACTCTGCCTTTCATCTACTGTGAAGCAGGTGGTACGCAGACTAA ATGAGCTGTACAAGGCCAGCGTGGTATCCTGCCAGGGCCTCAGCTTGCGACTTCAGCGCTTCT TTCTGGACAAACAACGGCTGCTGGACGGGATCCATGGTGTCACTGCAGAGCGGCTCATCCTCA GCCATGCTGTGCAAATGGTACAATCAGCTGCCCTTGATGAGATGTTCCAGCACCGAGAGGGCT GTGTACCGAGATATCACAAAGCCCTGCTATTGCTGGAGGGGTTGCAGCACACTCTCACGGACC AGGCAGACATTGAGAACATTGCCAAATGCAAGCTGTGCATTGAGAGGAGACTCTCGGCCCTGC TGAGTGGTGTCTATGCCTGA 

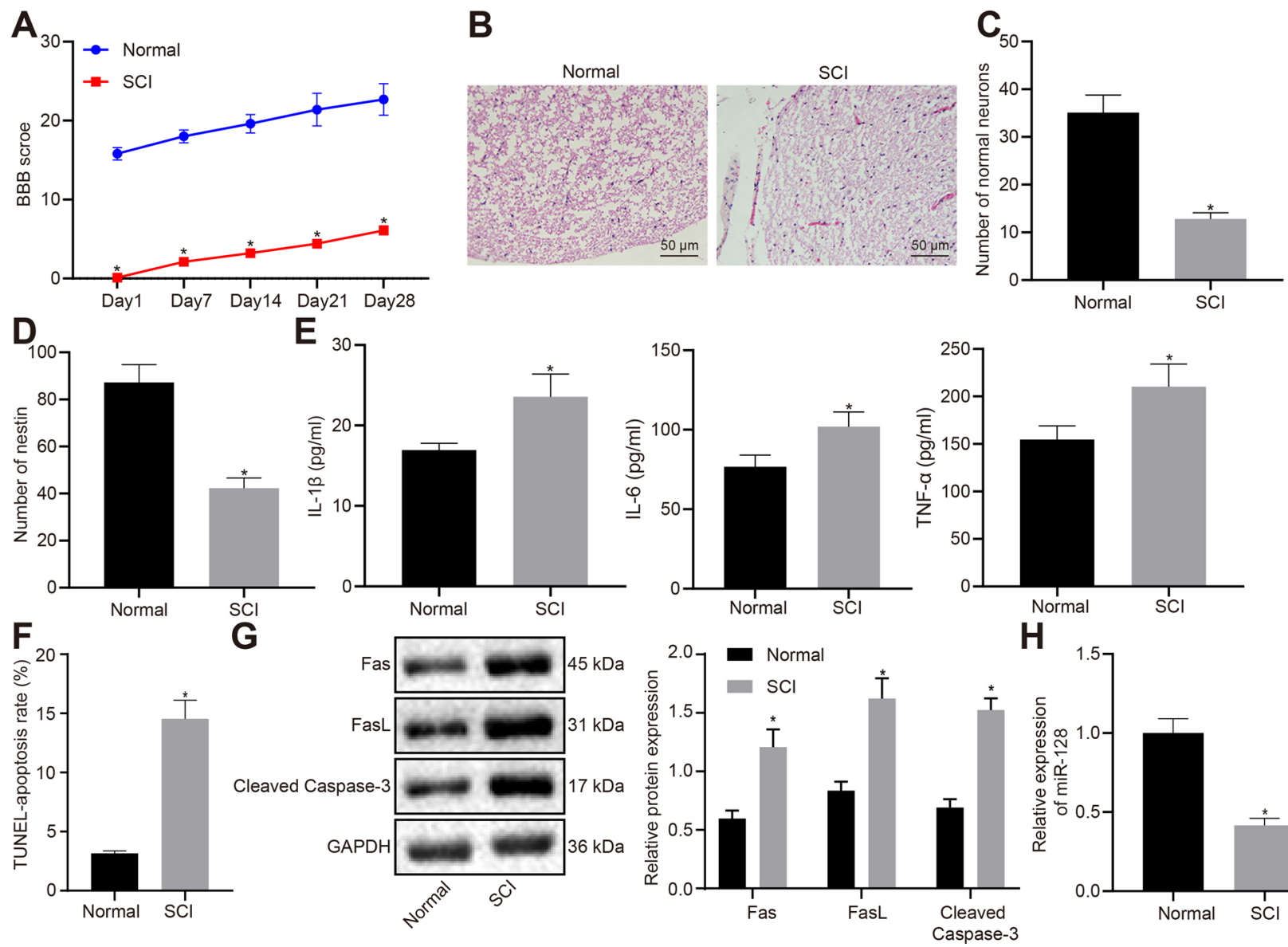

H

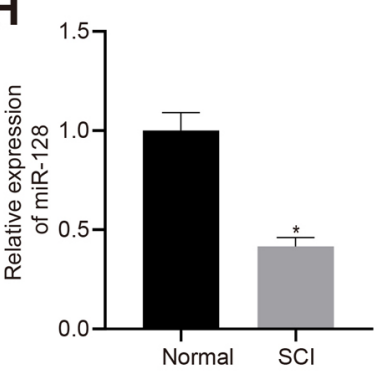

Figure 1. Rats with SCI exhibit increased neuronal apoptosis, intense inflammatory response and lowly expressed miR-128. (A) Locomotor functions of rats evaluated by BBB score. (B) Hematoxylin and eosin staining of morphological changes of neuronal cells (magnification, x200). (C) The survival of neurons in spinal cord of rats determined by Nissl staining. (D) The number of nestin-positive cells detected using immunofluorescence. (E) The expression of inflammatory factors (IL-1 $\beta$, IL-6 and TNF- $\alpha$ ) in rat serum determined by ELISA. (F) Apoptosis of spinal cord cells detected using TUNEL. (G) Protein expression of apoptosis-related factors measured by western blot analysis. $(\mathrm{H})$ The expression of miR-128 assessed by reverse transcription-quantitative PCR. An unpaired $t$-test was performed for comparisons of data between two groups. BBB score was used for comparison of data at different time points. Repeated-measures analysis of ANOVA was performed, followed by Bonferroni post-hoc test. "P<0.05 vs. normal. SCI, spinal cord injury; miR, microRNA; BBB, Basso, Beattie and Bresnahan; ANOVA, analysis of variance.

analysis demonstrated that the expression of apoptosis-related factors, Fas, FasL and cleaved caspase-3 rose markedly in rats with SCI compared with those in normal rats (Fig. 1G). The above results implied that neurons in rats with SCI exhibited promoted apoptosis, accompanied by inflammation. RT-qPCR (Fig. 1H) demonstrated that miR-128 expression in rats with SCI markedly declined compared with that in normal rats.

Overexpression of miR-128 attenuates SCI in rats. To confirm whether the changes in miR-128 expression affected the apoptosis and inflammatory response of rats with SCI, vectors including LV-control, LV-miR-128, or LV-sponge miR-128 were first constructed and injected into rats with SCI. As shown in Fig. 2A, RT-qPCR revealed that SCI rats injected with LV-sponge miR-128 exhibited markedly downregulated miR-128 levels, but an opposite trend was observed in SCI rats injected with LV miR-128 compared with SCI rats infected with LV-control $(\mathrm{P}<0.05)$. SCI Rats expressing LV-miR-128 showed a significantly higher BBB score while rats transfected with LV-sponge miR-128 showed significantly lower BBB scores in comparison with rats with SCI expressing LV-control $(\mathrm{P}<0.05$; Fig. 2B). Results from H\&E staining revealed that
SCI rats infected with LV-miR-128 showed alleviated pathological changes but SCI rats infected with LV-sponge miR-128 exhibited aggravated pathological changes when compared to rats infected with LV-control $(\mathrm{P}<0.05$; Fig. $2 \mathrm{C})$. The results of Nissl staining (Fig. 2D) and immunofluorescence (Fig. 2E) indicated that the number of normal neurons and nestin-positive cells of SCI rats injected with LV-miR-128 was significantly increased, but an opposite trend was observed in SCI rats injected with $\mathrm{LV}$-sponge miR-128 in comparison with rats injected with LV-control $(\mathrm{P}<0.05)$. The results of ELISA (Fig. 2F) indicated that the serum levels of IL-1 $\beta$, IL-6 and TNF- $\alpha$ in SCI rats infected with LV-miR-128 were significantly lower in comparison with those transduced with LV-control, whereas SCI rats infected with LV-sponge miR-128 showed a significant increase $(\mathrm{P}<0.05)$. TUNEL staining (Fig. 2G) demonstrated that SCI rats infected with LV-miR-128 showed a strikingly reduction in cell apoptosis while SCI rats infected with LV-sponge miR-128 showed a notable increase in apoptosis compared to rats injected with LV-control $(\mathrm{P}<0.05)$. Western blot analysis (Fig. 2H) demonstrated that Fas, FasL and cleaved caspase-3 expression in SCI rats infected with LV-miR-128 declined, while an opposite trend was observed 


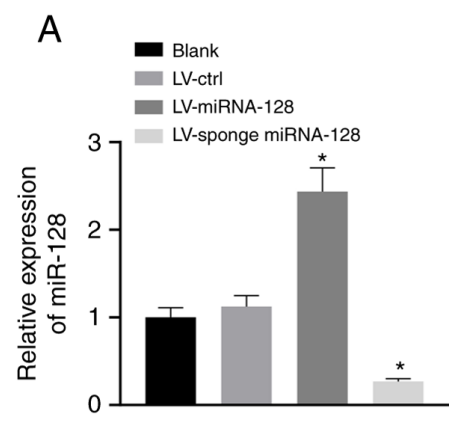

B

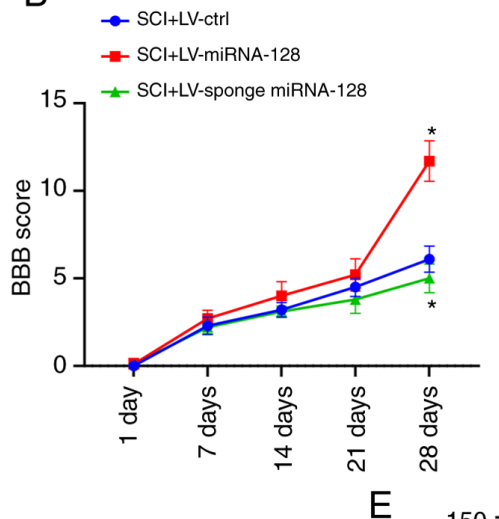

E
C

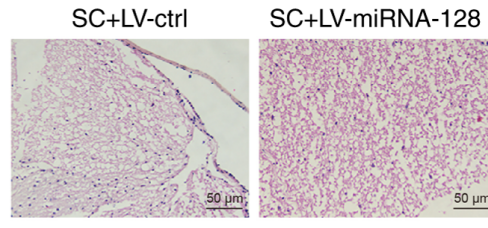

SC+LV-sponge miRNA-128

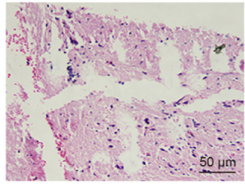

D

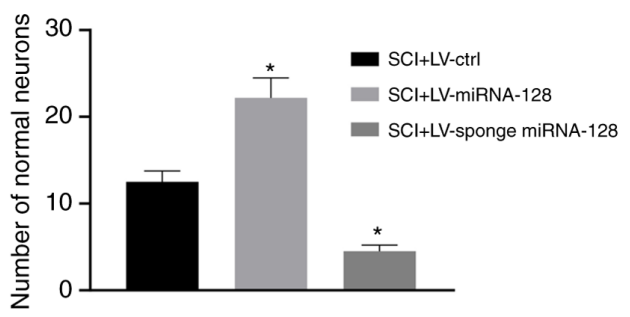

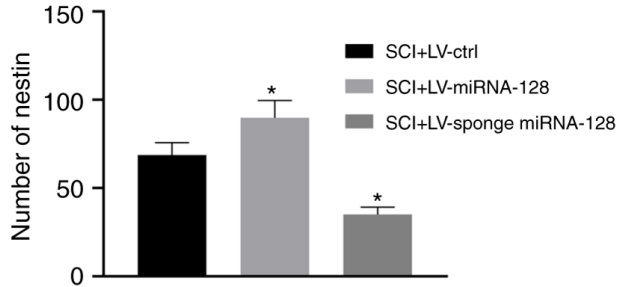

F

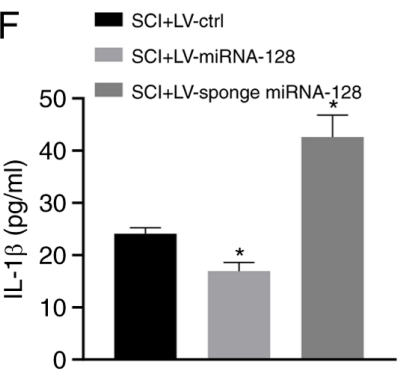

G

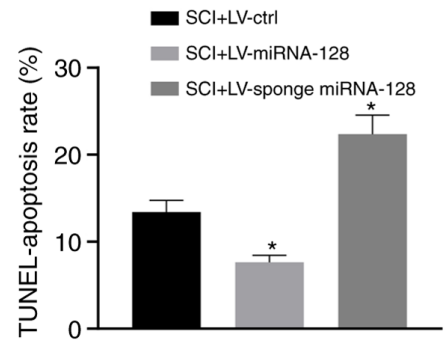

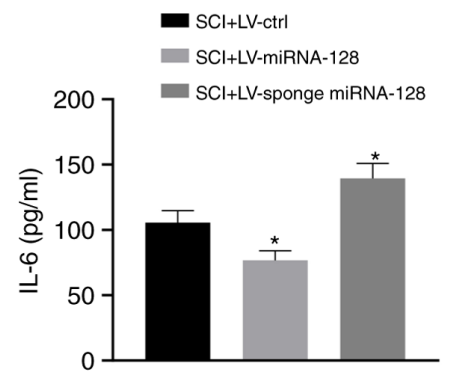

$\mathrm{H}$

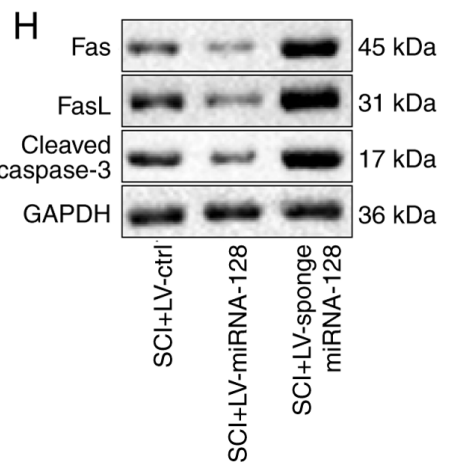

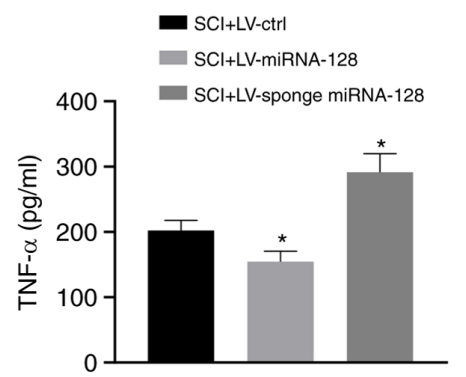

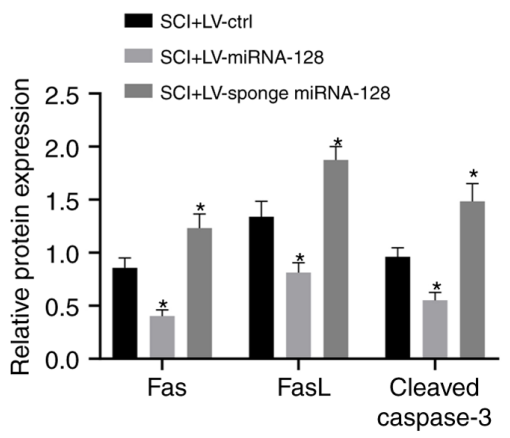

Figure 2. Upregulation of miR-128 can alleviate SCI in rats. Vectors containing LV-control, LV-miR-128, or LV-sponge miR-128 were injected into rats with SCI ( $\mathrm{n}=10$ /group). (A) miR-128 expression was assessed by reverse transcription-quantitative PCR. ${ }^{*} \mathrm{P}<0.05$ vs. SCI rats injected with LV-control. (B) Locomotor functions of rats indicated by BBB score. (C) Hematoxylin and eosin staining of morphological changes of neuronal cells (magnification, x200). (D) The survival of neuronal cells in rat spinal cord determined by Nissl staining. (E) The number of nestin-positive cells evaluated by immunofluorescence. (F) The levels of inflammatory factors in rat serum detected by ELISA. (G) Cell apoptosis was assessed by TUNEL staining. (H) The expression of apoptosis-related factor protein in rat spinal cord determined by western blot analysis. An unpaired $t$-test was performed for comparisons of data between two groups. ANOVA was performed for comparisons of data among multiple groups, followed by Tukey's post-hoc test. BBB score was used as the comparisons of different time points using repeated-measures analysis of ANOVA, followed by Bonferroni post-hoc test. "P<0.05 vs. SCI rats infected with LV-control. miR, microRNA; SCI, spinal cord injury; LV, Lentiviral vector; ctrl, control; BBB, Basso, Beattie and Bresnahan; ANOVA, analysis of variance.

in SCI rats infected with LV-sponge miR-128 in compared with those transduced with $\mathrm{LV}$-control $(\mathrm{P}<0.05)$. These above results suggested that the highly expressed miR-128 could inhibit apoptosis and the inflammatory response in SCI rats.

ULK1 is targeted by miR-128 and silencing of ULK1 relieves $S C I$ in rats. RNAInter, miRDB, TargetScan and miRWalk were employed to predict the target genes of miR-128. The results revealed that there were three genes appearing in the intersection, which consisted of ULK1, ABCB9 and SGMS1 (Fig. 3A). To further verify the predicted results, vectors containing LV-control, LV-miR-128, or LV-sponge miR-128 were injected into SCI rats. RT-qPCR and western blot analysis demonstrated that ULK1 expression was upregulated in rats 

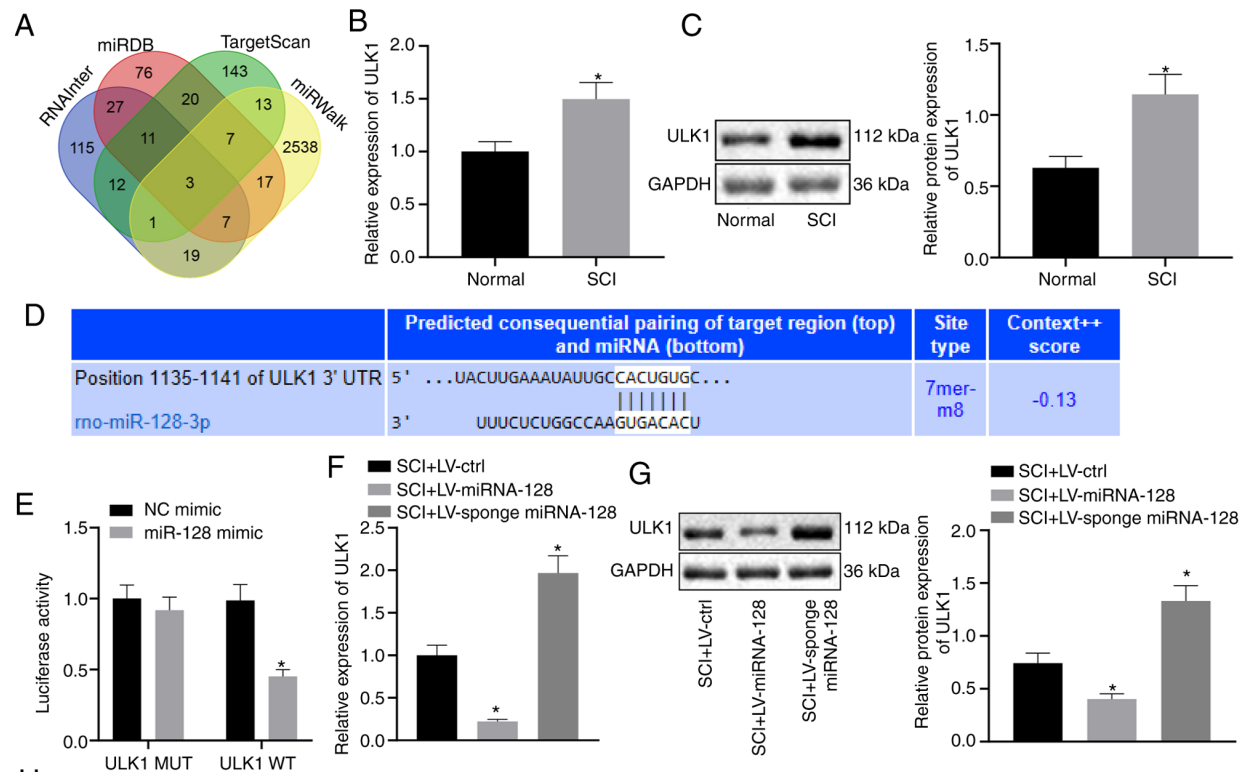

$F=\underset{S C l+L V-\text { miRNA-128 }}{\mathrm{SCl}} \quad \mathrm{G}$
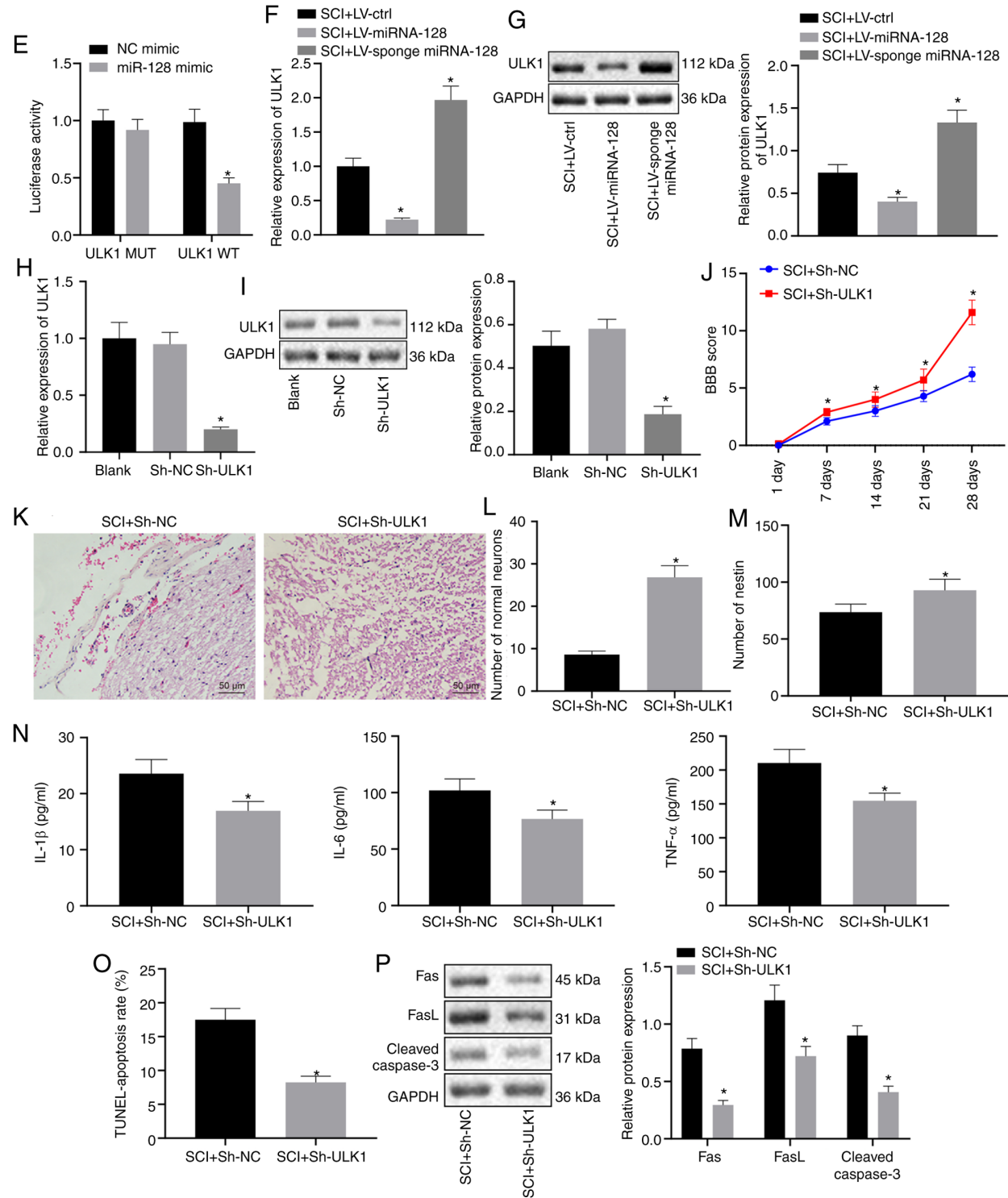

Figure 3. miR-128 inhibits the expression of ULK1 and knockdown of ULK1 attenuates SCI in rats. Vectors containing LV-control, LV-miR-128, or LV-sponge miR-128 were constructed and injected into rats with SCI in A-G, while vectors containing sh-NC or sh-ULK1 were injected into rats with SCI in H-P ( $n=10$ /group). (A) Venn diagram of the target genes of miR-128 predicted using RNAInter, miRDB, TargetScan and miRWalk. (B) The mRNA expression of ULK1 in normal rats and rats with SCI detected using RT-qPCR. ${ }^{*} \mathrm{P}<0.05$ vs. normal rats. (C) Protein expression of ULK1 detected by western blot analysis. ${ }^{\text {" }} \mathrm{P}<0.05$ vs. normal rats. (D) The binding site of miR-128 and ULK1 predicted using TargetScanHuman 7.2. (E) The targeting relationship between ULK1 and miR-128 measured by dual-luciferase report assay. ${ }^{~} \mathrm{P}<0.05$ vs. NC mimic. (F) The mRNA expression of ULK1 examined by RT-qPCR. "P $<0.05$ vs. SCI rats injected with LV-control. (G) The protein expression of ULK1 measured using western blot analysis. ${ }^{*} \mathrm{P}<0.05$ vs. SCI rats infected with LV-control. (H) The mRNA expression of ULK1 measured using RT-qPCR. "P<0.05 vs. Blank or sh-NC. (I) The protein expression of ULK1 expressing sh-NC and sh-ULK1 evaluated by western blot analysis. "P<0.05 vs. Blank or sh-NC. (J) Locomotor functions of rats evaluated by BBB score. (K) H\&E staining of morphological changes of neurons (magnification, x200). (L) Survival of neuronal cells detected by Nissl staining. (M) The number of nestin-positive cells detected by immunofluorescence. (N) The content of inflammatory factors in rat serum determined by ELISA. (O) Apoptosis of rat spinal cord cells determined using TUNEL. (P) The protein expression of apoptosis-related factor in rats with SCI evaluated by western blot analysis. J-P, "P $<0.05 \mathrm{vs}$. SCI rats transfected with sh-NC. An unpaired $t$-test was performed for comparisons of data between two groups. ANOVA was performed for comparisons of data among multiple groups, followed by Tukey's post-hoc test. BBB score was used as comparisons of data at different time points using repeated-measures analysis of ANOVA, followed by Bonferroni post-hoc test. miR, microRNA; ULK1, Unc-51 like autophagy activating kinase 1; SCI, spinal cord injury; RT-qPCR, reverse transcription-quantitative PCR; NC, negative control; LV, Lentiviral vector; ctrl, control; sh, short hairpin; ANOVA, analysis of variance. 
A

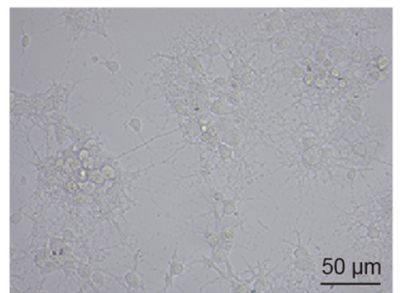

D
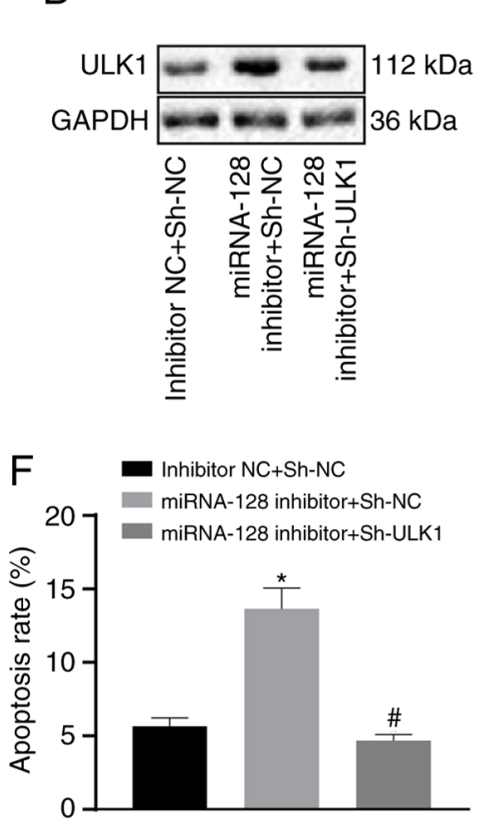

B

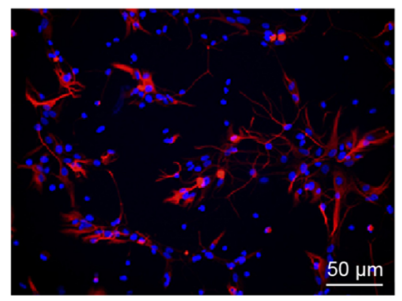

Inhibitor NC+Sh-NC
miRNA-128 inhibitor+Sh-NC
miRNA-128 inhibitor+Sh-ULK 1

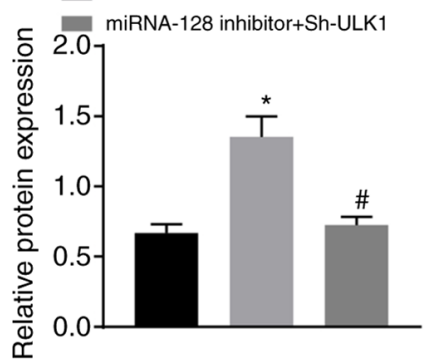

G

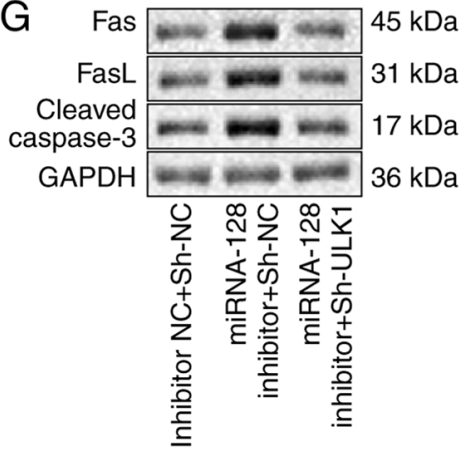

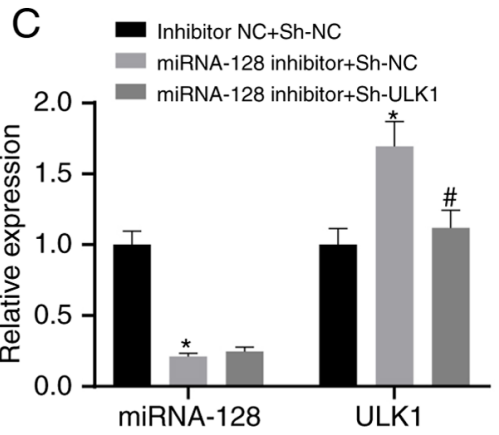

E $\rightarrow$ Inhibitor NC+Sh-NC
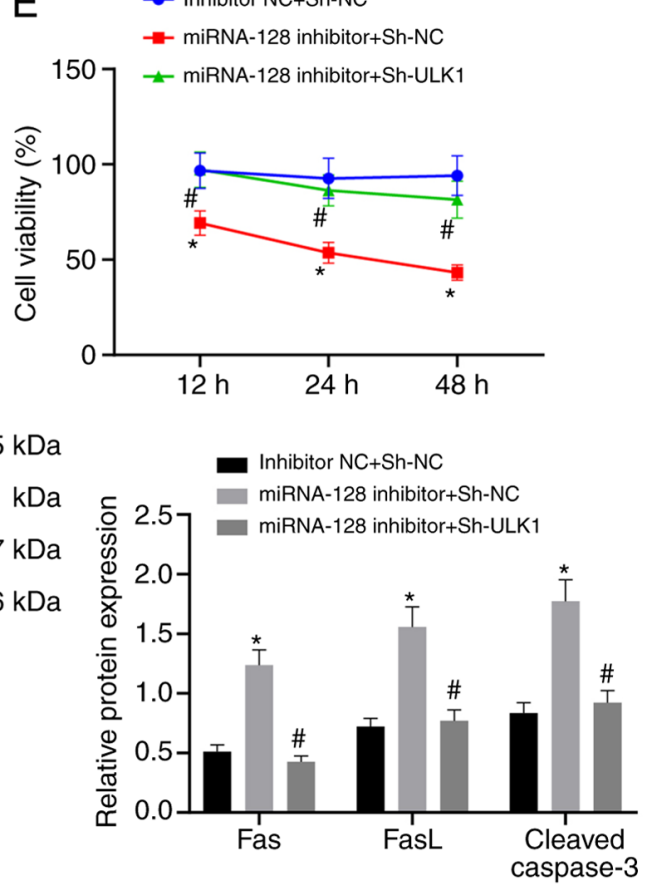
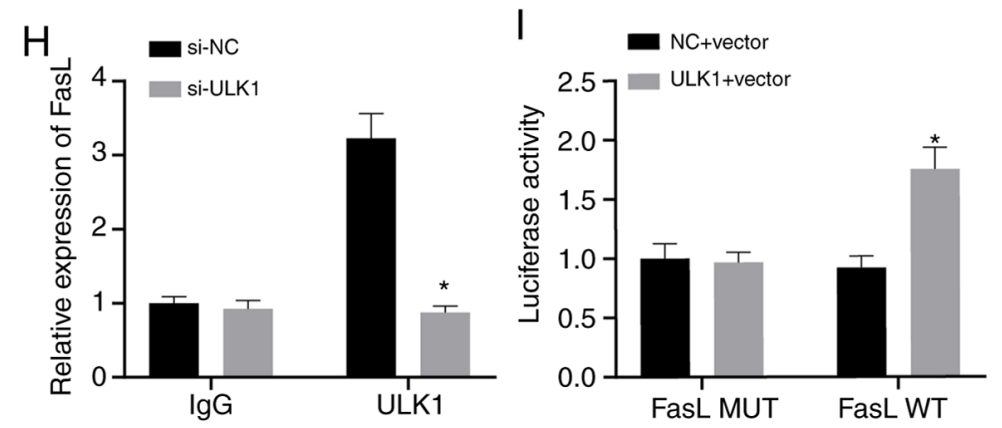

Figure 4. Upregulation of miR-128 repressed ULK1 expression and thereby attenuating SCI via the downregulation of FasL. The neuronal cells were transfected with inhibitor NC and sh-NC, miR-128 inhibitor and sh-NC or miR-128 inhibitor and sh-ULK1. (A) The morphology of spinal cord neurons observed under an inverted microscope (magnification, x200). (B) Neuronal cell nestin-positive expression determined by immunofluorescence (magnification, x200). (C) The expression of miR-128 and ULK1 detected using reverse transcription-quantitative PCR. (D) The protein expression of ULK1 measured by western blot analysis. (E) Cell proliferation assessed by MTT assay. (F) Cell apoptosis determined by flow cytometry. (G) The protein expression of apoptosis-related factors determined by western blot analysis. (H) The enrichment of ULK1 and FasL determined by ChIP assay. (I) The regulatory relationship of ULK1 on FasL assessed using dual-luciferase reporter assay. ${ }^{*} \mathrm{P}<0.05$ vs. cells co-transfected with inhibitor $\mathrm{NC}$ and sh-NC or ${ }^{\#} \mathrm{P}<0.05$ vs. cells co-transfected with miR-128 inhibitor and sh-NC, or cells co-transfected with si-NC. An unpaired $t$-test was performed for comparisons of data between two groups. ANOVA was conducted for comparison of data among multiple groups, followed by Tukey's post-hoc test. miR, microRNA; ULK1, Unc-51 like autophagy activating kinase 1; SCI, spinal cord injury; FasL, Fas ligand; NC, negative control; sh, short hairpin; WT, wild type; MUT, mutated; ChIP, Chromatin immunoprecipitation; IgG, immunoglobulin G.

with SCI ( $\mathrm{P}<0.05$; Fig. 3B and $\mathrm{C})$. The binding site between ULK1 and miR-128 was predicted using the bioinformatic website TargetScan Human version 7.2 (Fig. 3D). Results from dual-luciferase report assay confirmed that luciferase activity in the ULK1 WT was inhibited by miR-128 mimic, while
ULK1 MUT was not affected ( $\mathrm{P}<0.05$; Fig. 3E). Furthermore, RT-qPCR and western blot analysis indicated that ULK1 expression was markedly repressed in SCI rats infected with LV-miR-128 but was elevated in SCI rats injected with LV-sponge miR-128 ( $\mathrm{P}<0.05$; Fig. $3 \mathrm{~F}$ and $\mathrm{G})$. All these results 


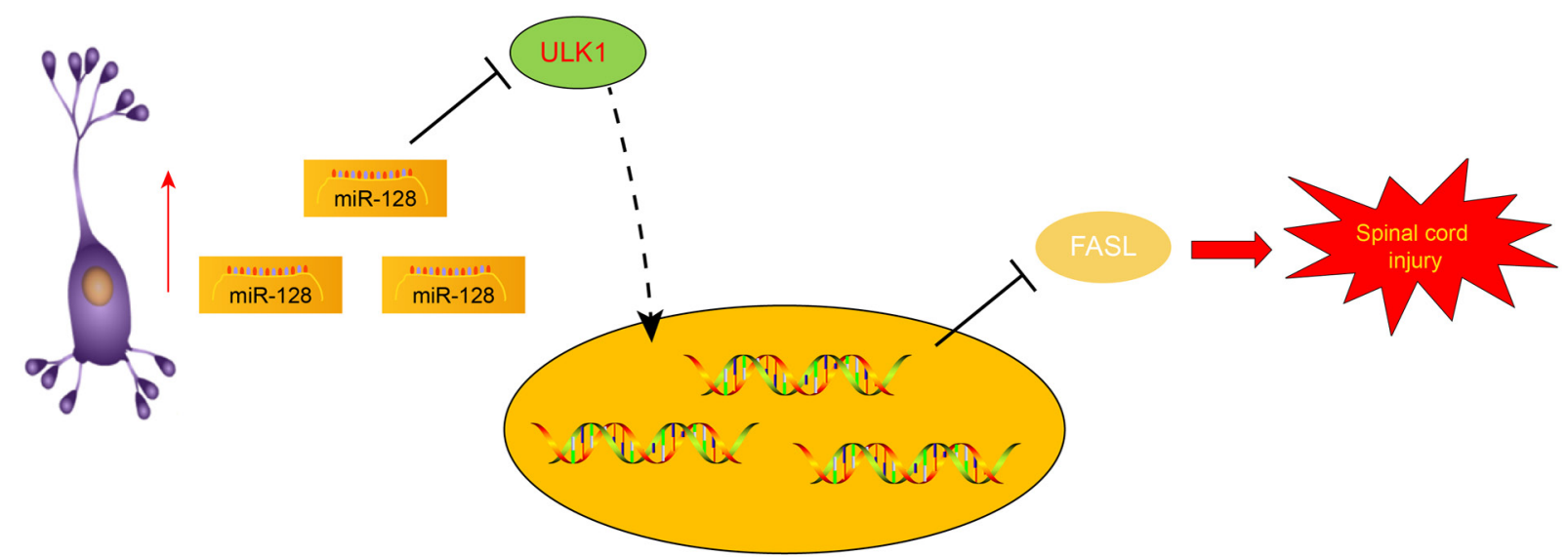

Figure 5. Molecular mechanism of miR-128/ULK1/FasL axis in protecting spinal cord injury. The upregulation of miR-128 could suppress ULK1 to inhibit the apoptosis of neuronal cells, thereby alleviating SCI via the downregulation of FasL. miR, microRNA; ULK1, Unc-51 like autophagy activating kinase 1; FasL, Fas ligand.

suggested that miR-128 could target and repress the expression of ULK1.

To further explore the effect of the changes in ULK1 expression on SCI rats, vectors containing sh-NC or sh-ULK1 was constructed and injected into SCI rats (Fig. $3 \mathrm{H}$ and I). Results showed that SCI rats transfected with sh-ULK1 exhibited significant higher BBB scores $(\mathrm{P}<0.05$; Fig. 3J). In addition, $\mathrm{H} \& \mathrm{E}$ staining revealed that $\mathrm{SCI}$ rats transfected with sh-ULK1 exhibited less severe pathological changes $(\mathrm{P}<0.05$; Fig. 3K).

The results from Nissl staining (Fig. 3L) and immunofluorescence (Fig. 3M) demonstrated that the number of normal neurons and nestin-positive cells were notably increased in SCI rats transfected with sh-ULK1 $(\mathrm{P}<0.05)$. ELISA demonstrated that the levels of IL-1 $\beta$, IL- 6 and TNF- $\alpha$ in SCI rats transfected with sh-ULK1 exhibited a notable decline $(\mathrm{P}<0.05$; Fig. 3N). The results of TUNEL (Fig. 3O) showed that reduced apoptosis was observed in SCI rats transfected with sh-ULK1 $(\mathrm{P}<0.05)$. Western blot analysis and the results showed that protein expression of Fas, FasL and Cleaved Caspase- 3 in SCI rats transfected with sh-ULK1 was significantly lowered $(\mathrm{P}<0.05$; Fig. 3P). Taken together, these results suggested that miR-128 could inhibit the expression of ULK1, thus relieving $\mathrm{SCI}$ in rats.

Elevation of miR-128 downregulates ULK1, thereby attenuating SCI via FasL repression in vitro. To verify the miR-128/ULK1 regulatory mechanism in vitro, fetal rat spinal cord neuronal cells were isolated and cultured. The isolated spinal cord neuronal cells began to adhere following 3-6 h of culture, accompanied by protruded cells. Following 2-3 d of culture, the cell began to bulge forcefully and the connection between cells became reticular. Spinal cord neuronal cells were successfully isolated and validated by immunofluorescence testing for nestin antibody staining (Fig. 4A and B). The neuronal cells were transfected with inhibitor $\mathrm{NC}+$ sh-NC, miR-128 inhibitor + sh-NC, or miR-128 inhibitor + sh-ULK1. RT-qPCR and western blot analysis (Fig. 4C and D) demonstrated that miR-128 expression in neuronal cells transfected with miR-128 inhibitor + sh-NC was significantly reduced, while the mRNA and protein expression of ULK1 was significantly increased in comparison with neuronal cells transfected with inhibitor $\mathrm{NC}+$ sh-NC. In addition, miR-128 expression in neuronal cells transfected with miR-128 inhibitor + sh-ULK1 remained unchanged, whereas mRNA and protein expression of ULK1 were both downregulated in comparison with those in neuronal cells transfected with miR-128 inhibitor + sh-NC. The results of MTT assay (Fig. 4E) demonstrated that the neuronal cell viability was markedly reduced by miR-128 inhibitor, which was negated by additional treatment with sh-ULK1. Flow cytometry demonstrated that the apoptosis rate of neuronal cells was augmented by miR-128 inhibitor, which was annulled by further sh-ULK1 treatment (Fig. 4F). As reflected by western blot analysis, the protein expression of Fas, FasL and cleaved caspase-3 was significantly enhanced in neuronal cells following miR-128 inhibitor treatment, which was abrogated by additional sh-ULK1 treatment (Fig. 4G). Meanwhile, the ChIP assay revealed that there was significant enrichment of ULK1 and FasL (Fig. 4H). Results from the dual-luciferase report assay showed that the cells transfected with oe-NC or oe-ULK1 had no significant difference in fluorescence intensity after co-transfection with FasL MUT. Compared with the cells transfected with oe-NC, cells transfected with oe-ULK1- and FasL WT exhibited an increase in fluorescence intensity (Fig. 4I). Together, the elevation of miR-128 could downregulate ULK1, thereby attenuating SCI via FasL downregulation.

\section{Discussion}

SCI causes permanent disruption and significantly destroys the central nervous system function, curbing the capability of patients to regenerate lost tissues (16). The differentiation of neural stem cells can contribute to neuronal differentiation, thereby attenuating SCI (17). A previous study demonstrated that the aberrant expression of miRNA following SCI has emerged as a potent research priority (18). The present study found evidence to support the hypothesis that the upregulation of miR-128 contributed to inhibited cell apoptosis and inflammation, as well as improved motor function following SCI via elevation of FasL by targeting and suppressing ULK1. 
Initially, the results of the present study demonstrated that miR-128 was poorly expressed in rats with SCI and neuronal cells from SCI. The overexpression of miR-128 resulted in inhibited neuronal cell apoptosis but contributed to improved motor function and inflammation, thereby alleviating SCI as evidenced by higher BBB score and abundant normal neurons and nestin-positive cells. However, it was also evidenced that the expression levels of IL- $1 \beta$, IL- 6 and TNF- $\alpha$, as well as protein expression of Fas, FasL and cleaved caspase-3, were notably decreased. A similar finding was mentioned in a previous study, whereby in conditioned medium-SCI-treated mouse microglia BV2, miR-128 expression is downregulated and upregulation of miR-128 contributes to an improved survival rate of microglia cells and reduces the concentration of inflammatory cytokines (TNF- $\alpha$, IL-1 $\beta$ and IL-6) (6).

In addition, miR-128 was associated with the development of the central nervous system and spinal cord neuroepithelial cells (19). Consistent with the results of the present study, SCI gives rise to increased neuronal apoptosis, resulting in irreversible neurological dysfunction of the spinal cord (20). The reduced neuronal death following SCI has been demonstrated to be positively correlated to improved motor function recovery (21). The inhibition of neuronal cell apoptosis has been shown to be favorable to protect the damaged spinal cord neurons (22). Increased expression of TNF- $\alpha$, IL-6 and IL-1 $\beta$ is indicative of a more severe degree of SCI, whereas the increased expression of apoptotic protein caspase-3 aggravates inflammation and apoptosis, which further results in repressed functional recovery (23). Fas/FasL signal transduction pathway is an important pathway to mediate cell apoptosis (24). The activation of the Fas/FasL system has been shown to contribute to ovarian cell apoptosis and impels oocyte capacity (25). Meanwhile, knockdown of FasL been shown to inhibit miR-21-induced apoptosis of keloid fibroblasts (26). In addition, an increased number of nestin-positive cells is indicative of attenuated SCI (27).

The results of previous study showed that ULK1 was negatively targeted by miR-128 and silencing of ULK1 resulted in attenuated SCI in rats, accompanied by inhibited neuronal cell apoptosis, improved motor function and inflammation, thereby alleviating SCI. These results were indicated by higher BBB score and more normal neurons, but notably decreased expression levels of IL-1 $\beta$, IL- 6 and TNF- $\alpha$, as well as protein expression of Fas, FasL, cleaved caspase-3. A previous study demonstrated that miR-372 can inhibit human pancreatic adenocarcinoma cell proliferation, migration, invasion, and autophagy by suppressing the expression of ULK1 (28). In addition, the overexpression of miR-26a-5p reduces ULK1 expression, whereas the inhibition of miR-26a-5p can elevate ULK1 expression (29). Absence of ULK1 results in an increased number of viable neurons in the hippocampus of traumatic brain injury in mice, thereby reducing nerve inflammation (7). The knockdown of ULK1 enhances the cell viability of neurons in a Parkinson's disease model (30).

In addition, the present study found that ULK1 positively regulated FasL expression and the downregulation of FasL contributed to attenuating SCI. Following the activation of hypoxic microglia, overexpression of FasL has been demonstrated to lead to neuronal apoptosis (31). miR-411 inhibits acute SCI by suppressing FasL expression in rats (32). Activation of
Fas/FasL has a significant function in mediating apoptosis, inflammatory response and neurodegeneration following SCI (11). Another study also noted that FasL expression is increased in acute SCI and its repression could attenuate acute SCI via downregulation in rats (32).

In conclusion, the present study revealed that the upregulation of miR-128 could suppress ULK1 to inhibit the apoptosis of neuronal cells, thereby alleviating SCI via the downregulation of FasL (Fig. 5). The current study provided further insights into the pathological mechanism of SCI and proposed a novel feasible option for the treatment of SCI. However, the potential molecular mechanism of miR-128 is expected to be implemented in the clinical setting in a future study.

\section{Acknowledgments}

Not applicable.

\section{Funding}

No funding was received.

\section{Availability of data and materials}

The datasets used and/or analyzed during the current study are available from the corresponding author on reasonable request.

\section{Authors' contributions}

YW and RLiu designed the study. ZP, YZ and RLi collated the data, carried out data analyses and produced the initial draft of the manuscript. YW and RLiu contributed to drafting the manuscript. ZP and YZ confirmed the authenticity of all the raw data. All authors have read and approved the final submitted manuscript.

\section{Ethics approval and consent to participate}

All animal experiments were implemented after ratification by the Animal Ethics Committee of the First Affiliated Hospital of Harbin Medical University (approval no. 2019010) conforming to Principles and Procedures of the National Academy of Animal Health Care Guidelines. All efforts were made to minimize animal suffering and the minimum number of animals used.

\section{Patient consent for publication}

Not applicable.

\section{Competing interests}

The authors declare that they have no competing interests.

\section{References}

1. Saxena T, Loomis KH, Pai SB, Karumbaiah L, Gaupp E, Patil K, Patkar R and Bellamkonda RV: Nanocarrier-mediated inhibition of macrophage migration inhibitory factor attenuates secondary injury after spinal cord injury. ACS Nano 9: 1492-1505, 2015. 
2. Ramer LM, Ramer MS and Bradbury EJ: Restoring function after spinal cord injury: Towards clinical translation of experimental strategies. Lancet Neurol 13: 1241-1256, 2014.

3. Assinck P, Duncan GJ, Hilton BJ, Plemel JR and Tetzlaff W: Cell transplantation therapy for spinal cord injury. Nat Neurosci 20: 637-647, 2017.

4. Zhang XM, Ma J, Sun Y, Yu BQ, Jiao ZM, Wang D, Yu MY, Li JY and Fu J: Tanshinone IIA promotes the differentiation of bone marrow mesenchymal stem cells into neuronal-like cells in a spinal cord injury model. J Transl Med 16: 193, 2018.

5. Ning B, Gao L, Liu RH, Liu Y, Zhang NS and Chen ZY: microRNAs in spinal cord injury: Potential roles and therapeutic implications. Int J Biol Sci 10: 997-1006, 2014.

6. Yang Z, Xu J, Zhu R and Liu L: Down-regulation of miRNA-128 contributes to neuropathic pain following spinal cord injury via activation of P38. Med Sci Monit 23: 405-411, 2017.

7. Wei HL, Ma SQ and Li CX: Deficiency of unc-51 like kinase 1 (Ulk1) protects against mice traumatic brain injury (TBI) by suppression of p38 and JNK pathway. Biochem Biophys Res Commun 503: 467-473, 2018.

8. Lu J, Zhu L, Zheng LP, Cui Q, Zhu HH, Zhao H, Shen ZJ, Dong HY, Chen SS, Wu WZ and Tan JM: Overexpression of ULK1 represents a potential diagnostic marker for clear cell renal carcinoma and the antitumor effects of SBI-0206965. EBioMedicine 34: 85-93, 2018.

9. Pang J, Han L, Liu Z, Zheng J, Zhao J, Deng K, Wang F and Zhang Y: ULK1 affects cell viability of goat Sertoli cell by modulating both autophagy and apoptosis. In Vitro Cell Dev Bio Anim 55: 604-613, 2019.

10. Krzyzowska M, Orlowski P, Baska P, Bodera P, Zdanowski R and Stankiewicz W: Role of Fas/FasL signaling in regulation of anti-viral response during HSV-2 vaginal infection in mice. Immunobiology 219: 932-943, 2014

11. Yu WR and Fehlings MG: Fas/FasL-mediated apoptosis and inflammation are key features of acute human spinal cord injury: Implications for translational, clinical application. Acta Neuropathol 122: 747-761, 2011.

12. Zhao CL, Cui HA and Zhang XR: MiR-543-5p inhibits inflammation and promotes nerve regeneration through inactivation of the NF-kB in rats after spinal cord injury. Eur Rev Med Pharmacol Sci 23 (3 Suppl): S39-S46, 2019.

13. von Leden RE, Khayrullina G, Moritz KE and Byrnes KR: Age exacerbates microglial activation, oxidative stress, inflammatory and NOX2 gene expression, and delays functional recovery in a middle-aged rodent model of spinal cord injury. J Neuroinflammation 14: 161, 2017.

14. Takenaga M, Ohta Y, Tokura Y, Hamaguchi A, Nakamura M, Okano H and Igarashi R: Lecithinized superoxide dismutase (PC-SOD) improved spinal cord injury-induced motor dysfunction through suppression of oxidative stress and enhancement of neurotrophic factor production. J Control Release 110: 283-289, 2006.

15. Livak KJ and Schmittgen TD: Analysis of relative gene expression data using real-time quantitative PCR and the 2(-Delta Delta C(T)) method. Methods 25: 402-408, 2001.

16. Li H, Ham TR, Neill N, Farrag M, Mohrman AE, Koenig AM and Leipzig ND: A hydrogel bridge incorporating immobilized growth factors and neural stem/progenitor cells to treat spinal cord injury. Adv Healthc Mater 5: 802-812, 2016.

17. Li X, Fan C, Xiao Z, Zhao Y, Zhang H, Sun J, Zhuang Y, Wu X, Shi J, Chen Y and Dai J: A collagen microchannel scaffold carrying paclitaxel-liposomes induces neuronal differentiation of neural stem cells through Wnt/ $\beta$-catenin signaling for spinal cord injury repair. Biomaterials 183: 114-127, 2018.
18. He J, Zhao J, Peng X, Shi X, Zong S and Zeng G: Molecular Mechanism of MiR-136-5p targeting NF-kB/A20 in the IL-17-mediated inflammatory response after spinal cord injury. Cell Physiol Biochem 44: 1224-1241, 2017.

19. Farrell BC, Power EM and Mc Dermott KW: Developmentally regulated expression of Sox 9 and microRNAs 124, 128 and 23 in neuroepithelial stem cells in the developing spinal cord. Int J Dev Neurosci 29: 31-36, 2011.

20. Shao Z, Lv G, Wen P, Cao Y, Yu D, Lu Y, Li G, Su Z, Teng P, Gao K, et al: Silencing of PHLPP1 promotes neuronal apoptosis and inhibits functional recovery after spinal cord injury in mice. Life Sci 209: 291-299, 2018.

21. Zheng G, Zhan Y, Wang H, Luo Z, Zheng F, Zhou Y, Wu Y, Wang S, Wu Y, Xiang G, et al: Carbon monoxide releasing molecule-3 alleviates neuron death after spinal cord injury via inflammasome regulation. EBioMedicine 40: 643-654, 2019.

22. Wang Z, Zhou L, Zheng X and Liu W: Effects of dexamethasone on autophagy and apoptosis in acute spinal cord injury. Neuroreport 29: 1084-1091, 2018.

23. Zhong ZX, Feng SS, Chen SZ, Chen ZM and Chen XW: Inhibition of MSK 1 promotes inflammation and apoptosis and inhibits functional recovery after spinal cord injury. J Mol Neurosci 68: 191-203, 2019

24. Wang M and Su P: The role of the Fas/FasL signaling pathway in environmental toxicant-induced testicular cell apoptosis: An update. Syst Biol Reprod Med 64: 93-102, 2018.

25. Li CY, Li ZB, Kong QQ, Han X, Xiao B, Li X, Chang ZL and Tan JH: Restraint-induced corticotrophin-releasing hormone elevation triggers apoptosis of ovarian cells and impairs oocyte competence via activation of the Fas/FasL system. Biol Reprod 99: 828-837, 2018.

26. Liu Y, Ren L, Liu W and Xiao Z: MiR-21 regulates the apoptosis of keloid fibroblasts by caspase- 8 and the mitochondria-mediated apoptotic signaling pathway via targeting FasL. Biochem Cell Biol 96: 548-555, 2018.

27. Lu PG, Hu SL, Hu R, Wu N, Chen Z, Meng H, Lin JK and Feng $\mathrm{H}$ : Functional recovery in rat spinal cord injury induced by hyperbaric oxygen preconditioning. Neurol Res 34: 944-951, 2012.

28. Chen H, Zhang Z, Lu Y, Song K, Liu X, Xia F and Sun W: Downregulation of ULK1 by microRNA-372 inhibits the survival of human pancreatic adenocarcinoma cells. Cancer Sci 108: 1811-1819, 2017.

29. Zheng L, Lin S and Lv C: MiR-26a-5p regulates cardiac fibroblasts collagen expression by targeting ULK1. Sci Rep 8: 2104, 2018.

30. Li Y, Zhang J and Yang C: UNC-51-like kinase 1 blocks S6k1 phosphorylation contributes to neurodegeneration in Parkinson's disease model in vitro. Biochem Biophys Res Commun 459: 196-200, 2015.

31. Zhang L, Dong LY, Li YJ, Hong Z and Wei WS: miR-21 represses FasL in microglia and protects against microglia-mediated neuronal cell death following hypoxia/ischemia. Glia 60: 1888-1895, 2012

32. Gong ZM, Tang ZY and Sun XL: miR-411 suppresses acute spinal cord injury via downregulation of Fas ligand in rats. Biochem Biophys Res Commun 501: 501-506, 2018.

This work is licensed under a Creative Commons Attribution-NonCommercial-NoDerivatives 4.0 International (CC BY-NC-ND 4.0) License. 